We are now in a position to take up the proof of Theorem III. Clearly $\varphi(u / v, u / v)$ is equal to $J_{\phi}(u, v) / K_{\phi}(u, v)$. Let $u$ and $v$ be two numbers such that $J_{\phi}(u, v)$ and $K_{\phi}(u, v)$ are prime to one another. By lemma 1, such numbers exist. Then to say that

$$
J_{\phi}(u, v) / K_{\phi}(u, v) \equiv u / v\left(\bmod p^{k}\right)
$$

is equivalent to saying

$$
L_{\phi}(u, v) \equiv 0\left(\bmod p^{k}\right),
$$

or that $p^{k}$ is a factor of $L_{\phi}(u, v)$. If $\varphi$ is iteratively equivalent to $\psi$, clearly we must have

$$
L_{\psi}(u, v) \equiv 0\left(\bmod p^{k}\right) .
$$

That is, every prime power that divides $L_{\phi}$ must also divide $L_{\psi}$, or in other words, $L_{\phi}$ is a factor of $L_{\psi}$ for a particular set of values of $u$ and $v$. However, since by Theorem I the equations $L_{\phi}(u, 1)=0$ and $L_{\psi}(u, 1)=0$ have the same three distinct roots, $L_{\psi}$ is a constant multiple of $L_{\phi}$. Hence $L_{\phi}$ is always a factor of $L_{\psi}$. Likewise, $L_{\psi}$ is always a factor of $L_{\phi}$. Hence, apart from a possible difference of sign, $L_{\phi}$ and $L_{\psi}$ are identical. This proves Theorem III.

Massachusetts Institute of Technology, April 16, 1920.

\title{
NECESSARY AND SUFFICIENT CONDITIONS THAT A LINEAR TRANSFORMATION BE COMPLETELY CONTINUOUS.
}

BY PROFESSOR CHARLES ALBERT FISCHER.

(Read before the American Mathematical Society December 31, 1919.)

A LARGE part of the Fredholm theory of integral equations has been derived for the equation

$$
h(x)=f(x)+\lambda T(f),
$$

where $T(f)$ is a completely continuous linear transformation.* It has also been proved that every linear transformation, that

* F. Riesz, Acta Mathematica, vol. 41 (1918), pp. 71-98. 
is, linear functional depending on a parameter, is equal to a Stieltjes integral of the form*

$$
T(f)=\int_{a}^{b} f(y) d_{y} K(x, y) .
$$

In a former paper $\dagger$ I have found a necessary and some sufficient conditions that a linear transformation be completely continuous, and thus proved that this part of the Fredholm theory applies to Stieltjes integral equations of certain types. In the present paper will be found conditions which are both necessary and sufficient. The Volterra theory of Stieltjes integral equations has been discussed by Evans in his Cambridge Colloquium Lectures, 1916.

\section{§1. Preliminary Theorems.}

A set of functions is said to be compact if every infinite sequence of them contains an infinite subsequence which is uniformly convergent, and a transformation is completely continuous if it changes every bounded set of functions into a compact set.

The class $[f(y)]$ will be composed of all functions defined on the interval $(a, b)$ which can be approached by monotone sequences, beginning with the class of all continuous functions. The definition of the Stieltjes integral has been extended by Young to apply to such functions.

The function $K(x, y)$ will be assumed to satisfy the equation

$$
K(x, y)=K(x, y+0) \text {. }
$$

This will not affect the value of the integral (1), and when this equation is satisfied, $V_{y} K(x, y)$, that is, the variation of $K$ considered as a function of $y$, is the least upper bound of $T(f) / \max |f| . \S \quad$ It follows that if $V_{y} K(x, y)$ is not bounded uniformly in $x$, the transformation (1) cannot be completely continuous, because in that case there would be a bounded set of $f$ 's for which the set $[T(f)]$ would not be bounded and therefore could not be compact.

* F. Riesz, Annales Scientifiques de l'Ecole Normale, ser. 3, vol. 31 (1914), pp. $9-14$.

$\dagger$ This Bulletin, vol. 25 (1919), p. 447.

¥ Young, Proc. London Math. Society, vol. 13 (1914), p. 109, or Daniell, Annals of Mathematics; vol. 19 (1918), p. 279.

§Riesz, loc. cit., Annales; or Fischer, Annals of Mathematics, vol. 19 (1917), pp. 38-40. 
It will now be proved that when $V_{y} K$ is bounded uniformly, every sequence of $x$ 's must contain a subsequence for which $K\left(x_{n}, y\right)$ approaches a uniquely determined function of $y$ when $n$ becomes infinite.

The positive constants $\epsilon_{1}, \epsilon_{2}, \ldots$ will be chosen so as to approach zero as a limit, and it will be assumed that $V_{y} K$ $<M$. If there were a sequence of $x$ 's no subset of which would make the limit of $K\left(x_{n}, y\right)$ unique, there would be no subset which would make the difference between the largest and smallest limit points of $K\left(x_{n}, y\right)$ less than or equal to $\epsilon_{1}$ uniformly in $y$, or else there would be such a subset which will be designated as $x_{1}{ }^{(1)}, x_{2}{ }^{(1)}, \ldots$. Then there would be no subset of the $x^{(1)}$ 's which would make the same difference less than $\epsilon_{2}$ uniformly, or else there would be such a subsequence, $x_{1}{ }^{(2)}, x_{2}{ }^{(2)}, \cdots$. It would not be possible to proceed in this way and get an infinite number of sequences, $x_{1}{ }^{(i)}$, $x_{2}{ }^{(i)}, \cdots$, each a subset of the proceeding, and such that the difference between the largest and smallest limit points of $K\left(x_{n}{ }^{(i)}, y\right)$ would always be less than $\epsilon_{i}$, because if this could be done the limit of $K\left(x_{n}{ }^{(n)}, y\right)$ would be unique. Consequently there would have to be a subset of the original $x$ 's, $\bar{x}_{1}, \bar{x}_{2}, \cdots$, and a $k>0$, such that no subsequence of the $\bar{x}$ 's could make the difference between the largest and smallest limit points of $K\left(\bar{x}_{n}, y\right)$ less than or equal to $k$ uniformly in $y$.

When a bounded set of functions is given there is always a subset of them that converges at any previously given denumerable set of points. ${ }^{*}$ It follows that there would be a nondenumerable set of values of $y$ for which the difference between limit points of $K\left(\bar{x}_{n}, y\right)$ would be greater than $k$, for a given sequence of the $\bar{x}$ 's, since if there were only a denumerable set of such $y$ 's, the subset of the $\bar{x}$ 's which made the above limit unique for these $y$ 's would make the difference between limit points of $K\left(\bar{x}_{n}, y\right)$ less than or equal to $k$ for all values of $y$.

A subset of the $\bar{x}$ 's could be taken such that $K\left(\bar{x}_{n}, y\right)$ would converge at a dense set of $y$ 's, and the other $\bar{x}$ 's dropped. Then if $y_{1}$ were a point where two of its limit points differed by more than $k$, there would be two $\bar{x}$ 's which would make $K$ differ by more than $k$ at $y_{1}$, while they made it differ by an arbitrarily small amount at a point arbitrarily near to $y_{1}$, and consequently $V_{y} K$ would be greater than $k / 2$ in the neigh-

* Riesz, loc. cit., Acta, p. 93. 
borhood of $y_{1}$ for at least one of these values of $x$. To state this more definitely, if a sequence $\delta_{1}, \delta_{2}, \ldots$, were given, approaching zero, there would be a sequence of $\bar{x}$ 's which would satisfy the inequalities

$$
V_{y} K\left(\bar{x}_{n}, y\right) \mid \begin{aligned}
& y_{1}+\delta_{n} \\
& y_{1}-\delta_{n}
\end{aligned}>\frac{k}{2} \quad(n=1,2, \cdots) .
$$

The other $\bar{x}$ 's could then be dropped. Similarly there would be a $y_{2}$ and a subset of the remaining $\vec{x}$ 's which would have the same property with respect to $y_{2}$, and the other $\bar{x}$ s could again be dropped. Proceeding in this way, the points $y_{1}, y_{2}$, $\cdots, y_{N}(N>2 M / k)$ and a sequence of $\bar{x}$ 's would be determined which would satisfy the inequalities

$$
V_{y} K\left(\bar{x}_{n}, y\right) \mid \begin{aligned}
& y_{i}+\delta_{n} \\
& y_{i}-\delta_{n}
\end{aligned}>\frac{k}{2} \quad(i=1,2, \cdots, N) .
$$

If $n$ were then taken so large that the intervals $\left(y_{i}-\delta_{n}\right.$, $y_{i}+\delta_{n}$ ) did not overlap, the inequalities

$$
V_{y} K\left(\bar{x}_{n}, y\right) \geqq \sum_{i=1}^{N} V_{y} K\left(\bar{x}_{n}, y\right) \mid \begin{aligned}
& y_{i}+\delta_{n} \\
& y_{i}-\delta_{n}
\end{aligned}>\frac{N k}{2}>M,
$$

would be satisfied. But this is contrary to the hypothesis that $V_{y} K<M$.

This completes the proof of the theorem: If $V_{y} K$ is bounded uniformly in $x$, when a denumerable set of the $x$ 's is given, there must be a subsequence of them which makes $K\left(x_{n}, y\right)$ approach a unique limit as $n$ becomes infinite.

It will now be proved that if a set of functions converges, the variation of the limiting function cannot be greater than the limit of the variations of any sequence of the given functions.

If this were not the case there would be a sequence $\varphi_{1}(y)$, $\varphi_{2}(y), \cdots$, approaching a function $\varphi(y)$, and a $k>0$, which would satisfy the inequalities

$$
V \varphi(y)>V \varphi_{n}(y)+k \quad(n=1,2, \cdots) .
$$

Then there would have to be a finite set of points $y_{0}=a$ $<y_{1}<y_{2}<\cdots<y_{N}=b$, which would satisfy the inequalities

$$
\sum_{i=0}^{N-1}\left|\varphi\left(y_{i+1}\right)-\varphi\left(y_{i}\right)\right|>V \varphi_{n}(y)+\frac{k}{2} \quad(n=1,2, \cdots)
$$


But if $n$ is large enough the inequalities

$$
\left|\varphi_{n}\left(y_{i}\right)-\varphi\left(y_{i}\right)\right|<\frac{k}{4 N} \quad(i=0,1,2, \cdots, N),
$$

would be satisfied, and consequently inequality (3) would imply that

$$
\sum_{i=0}^{N-1}\left|\varphi_{n}\left(y_{i+1}\right)-\varphi_{n}\left(y_{i}\right)\right|>V \varphi_{n}(y)
$$

which is absurd.

\section{§2. Necessary and Sufficient Conditions.}

The following condition, which will be called condition $A$, will be proved to be necessary. Then a second condition, which will be called condition $B$, will be proved to be sufficient, and finally the two conditions will be proved to be equivalent.

Condition $A . \quad A$ necessary and sufficient condition that the transformation (1) be completely continuous is that $V_{y} K(x, y)$ shall be bounded uniformly, and that when a sequence of $x$ 's is chosen in such a way that $K\left(x_{n}, y\right)$ converges, the equation

$$
\operatorname{limit}_{n=\infty} V_{y}\left[K\left(x_{n}, y\right)-\operatorname{limit}_{n=\infty} K\left(x_{n}, y\right)\right]=0
$$

shall be satisfied.

If this condition is not satisfied there must be a sequence of $x$ 's for which $K\left(x_{n}, y\right)$ converges, and a $k>0$, which satisfy the inequalities

$$
V_{y}\left[K\left(x_{n}, y\right)-\operatorname{limit}_{n=\infty} K\left(x_{n}, y\right)\right]>k .
$$

If the functions $K\left(x_{n}, y\right)-\operatorname{limit}_{n=\infty} K\left(x_{n}, y\right)$ should also satisfy equations such as (2), there would be continuous functions $f_{1}(y), f_{2}(y), \cdots$, not greater than 1 in absolute value, which would satisfy the inequalities

$$
\begin{aligned}
\int_{a}^{b} f_{m}(y) d_{y}\left[K\left(x_{m}, y\right)-\operatorname{limit}_{n=\infty} K\left(x_{n}, y\right)\right] & >k \\
(m & =1,2, \cdots) .
\end{aligned}
$$

Since this is not necessarily the case, it will be proved instead that when $x_{m}$ is given there must be an $m^{\prime} \geqq m$, and a con- 
tinuous $f_{m}(y)$, such that $\left|f_{m}\right| \leqq 1$, and

$$
\int_{a}^{b} f_{m}(y) d_{y}\left[K\left(x_{m}, y\right)-\operatorname{limit} K\left(x_{n}, y\right)\right]>\frac{k}{2} .
$$

It follows from the last theorem in $\S 1$, that when inequalities (5) are satisfied, when $m$ is given there must be an $n>m$ which satisfies the inequality

$$
V_{u}\left[K\left(x_{m}, y\right)-K\left(x_{n}, y\right)\right]>k .
$$

Consequently there must be an $f_{m}(y)$ which satisfies the inequality

$$
\int_{a}^{b} f_{m}(y) d_{y}\left[K\left(x_{m}, y\right)-K\left(x_{n}, y\right)\right]>k,
$$

and at least one of the points $x_{m}$ and $x_{n}$ can be used for $x_{m^{\prime}}$, in inequality (6).

The equation

$$
\operatorname{limit}_{n=\infty} \int_{a}^{b} f(y) d_{y} K\left(x_{n}, y\right)=\int_{a}^{b} f(y) d_{y} \operatorname{limit} K\left(x_{n}, y\right)
$$

must be satisfied if $f(y)$ is continuous.* Then when $f_{m}(y)$ is given there must be a finite $N$ such that

$\left|\int_{a}^{b} f_{m}(y) d_{y}\left[K\left(x_{n}, y\right)-\operatorname{limit} K\left(x_{n}, y\right)\right]\right|<\frac{k}{6} \quad(n \geqq N)$.

It will then be possible to select a sequence $\xi_{1}, \xi_{2}, \ldots$ from the given $x$ 's, and a sequence of continuous $f$ 's, which satisfy the inequalities, $\left|f_{i}\right| \leqq 1$, and

and

$$
\begin{aligned}
\left|\int_{a}^{b} f_{i}(y) d_{y}\left[K\left(\xi_{j}, y\right)-\operatorname{limit} K\left(x_{n}, y\right)\right]\right|<\frac{k}{6} \\
(i=1,2, \cdots ; j=i+1, i+2, \cdots),
\end{aligned}
$$

$$
\int_{a}^{b} f_{j}(y) d_{y}\left[K\left(\xi_{j}, y\right)-\operatorname{limit} K\left(x_{n}, y\right)\right]>\frac{k}{2}
$$$$
(j=1,2, \cdots) \text {. }
$$

* This follows from the proof of Bray's Theorem 3, Annals of Mathematics, ser. 2, vol. 20, pp. 180-181. 
This implies that either

$$
\left|\int_{a}^{b}\left[f_{j}(y)-f_{i}(y)\right] d_{y} K\left(\xi_{j}, y\right)\right|>\frac{1}{2}\left(\frac{k}{2}-\frac{k}{6}\right)=\frac{k}{6},
$$

or else

$$
\mid \int_{a}^{b}\left[f_{j}(y)-f_{i}(y)\right] d_{y} \text { limit } K\left(x_{n}, y\right) \mid>\frac{k}{6} .
$$

In either case there must be some value of $x$ for which

$$
\begin{aligned}
& \left|T\left(f_{j}\right)-T\left(f_{i}\right)\right|>\frac{k}{6} \\
& \quad(i=1,2, \cdots ; j=i+1, i+2, \cdots),
\end{aligned}
$$

and the transformation cannot be completely continuous. Therefore condition $A$ is necessary.

Condition B. A necessary and sufficient condition that the transformation (1) be completely continuous, is that when an $\epsilon>0$ is chosen there must be a finite number of points $\xi_{1}, \xi_{2}$, $\cdots, \xi_{n}$, such that to every $x$ in $(a, b)$ there corresponds a $\xi_{i}$ which satisfies the inequality

$$
V_{y}\left[K(x, y)-K\left(\xi_{i}, y\right)\right]<\epsilon .
$$

If this condition is satisfied, and a decreasing sequence $\mathfrak{\epsilon}_{1}, \epsilon_{2}, \cdots$ is taken approaching zero, the $\xi$ 's corresponding to all the $\epsilon$ 's can be arranged in one denumerable sequence. Every bounded set of $f$ 's must have a subset such that the functions

$$
g_{n}(x)=T\left(f_{n}\right)=\int_{a}^{b} f_{n}(y) d_{y} K(x, y) \quad(n=1,2, \cdots),
$$

will converge at the denumerable set of points $\xi_{1}, \xi_{2}, \ldots$. This subset will now be proved uniformly convergent on the whole interval $(a, b)$.

If an $\epsilon>0$ is chosen there must be a finite $N$ such that to every $x$ on $(a, b)$ there will correspond a $\xi_{i},(i \leqq N)$, which satisfies inequality (7). Then if $N^{\prime}$ is so large that the inequalities

$$
\left|g_{n}\left(\xi_{i}\right)-\operatorname{limit}_{n=\infty} g_{n}\left(\xi_{i}\right)\right|<\epsilon \quad\left(i \leqq N ; n \geqq N^{\prime}\right),
$$


1920.] ROOTS AND POLES OF RATIONAL FUNCTIONS.

are satisfied, the inequalities

$$
\left|g_{n}(x)-g_{n}\left(\xi_{i}\right)\right| \leqq \max |f| \cdot V_{y}\left[K(x, y)-K\left(\xi_{i}, y\right)\right]
$$

and inequality (7) will imply that

$$
(n=1,2, \cdots) \text {, }
$$

$$
\begin{aligned}
\left|g_{n}(x)-g_{n+m}(x)\right| \leqq 2 & {[\max |f|+1] \epsilon } \\
& \left(n \geqq N^{\prime} ; m=1,2, \cdots\right) .
\end{aligned}
$$

Consequently these $g$ 's must be uniformly convergent, and the transformation must be completely continuous.

If condition $B$ is not satisfied there must be an $\epsilon>0$ and an infinite sequence of $x$ 's such that

$$
V_{y}\left[K\left(x_{m}, y\right)-K\left(x_{n}, y\right)\right] \geqq \epsilon \quad(m \neq n) .
$$

It follows from the principal theorem of the first section, that if $V_{y} K$ is bounded uniformly, a subset of these $x$ 's must make $K\left(x_{n}, y\right)$ convergent. As no subset of them can satisfy equation (4), condition $A$ cannot be satisfied. Therefore when $A$ is satisfied $B$ must be, and since $A$ is necessary and $B$ sufficient, $B$ must be necessary and $A$ sufficient also.

Trinity College, HartFord, Conn.

\section{ON THE RELATION OF THE ROOTS AND POLES OF A RATIONAL FUNCTION TO THE ROOTS OF ITS DERIVATIVE.}

BY MR. BEN-ZION LINFIELD.

(Read before the American Mathematical Society December 30, 1919.)

1. F. Lucas, Journal de l'Ecole polytechnique, 1879, gave a mechanical proof of the following theorem:

The roots of the derivative of a cubic are the foci of the maximum ellipse inscribed in the triangle whose vertices are the roots of the cubic.

Professor Maxime Bôcher gave a simple proof of this theorem, Annals of Mathematics, volume 7, page 70, 1892. Here he made observations on the general theorem concerning the polynomial of $n$th degree and asked the question, "Could 